\title{
Multiplex limited penetrable horizontal visibility graph from EEG signals for driver fatigue detection
}

\author{
Qing Cai, Zhong-Ke Gao*, Yu-Xuan Yang, Wei-Dong Dang \\ School of Electrical and Information Engineering, Tianjin University \\ No.92, Weijin Road,Nankai District \\ Tianjin, 300072, China \\ zhongkegao@tju.edu.cn \\ Celso Grebogi \\ Institute for Complex Systems and Mathematical Biology, Kings College, University of Aberdeen \\ Aberdeen AB24 3UE, UK
}

\begin{abstract}
Driver fatigue is an important contributor to road accidents, and driver fatigue detection has attracted a great deal of attentions on account of its significant importance. Numerous methods have been proposed to fulfill this challenging task, though, the characterization of the fatigue mechanism still, to a large extent, remains to be investigated. To address this problem, we in this work, develop a novel Multiplex Limited Penetrable Horizontal Visibility Graph (Multiplex LPHVG) method, which allows not only detecting fatigue driving but also probing into the brain fatigue behavior. Importantly, we use our method to construct brain networks from EEG signals recorded from different subjects performing simulated driving tasks under alert and fatigue driving states. We then employ clustering coefficient, global efficiency and characteristic path length to characterize the topological structure of the networks generated from different brain states. In addition, we combine average edge overlap with the network measures to distinguish alert and mental fatigue states. The high-accurate classification results clearly demonstrate and validate the efficacy of our multiplex LPHVG method for the fatigue detection from EEG signals. Furthermore, our findings show a significant increase of the clustering coefficient as the brain evolves from alert state to mental fatigue state, which yields novel insights into the brain behavior associated with fatigue driving.
\end{abstract}

Keywords: Multiplex limited penetrable horizontal visibility graph; EEG; brain network; driver fatigue detection.

\section{Introduction}

Mental fatigue refers to suboptimal psychophysiological condition caused by prolonged periods of demanding cognitive activity. ${ }^{1}$ Driver fatigue is a state of reduced mental alertness, which impairs performance of driving, being a major contributing factor for traffic accidents globally. ${ }^{2}$ As a consequence, developing and establishing new technologies to monitor and predict driver's mental state contribute significantly to reduce road accidents and improve the health of drivers and passengers.

Many countermeasures against driver fatigue have been proposed, including driver physiological signals measuring and driver behavior monitoring. It is difficult to assess the exact mental state only from the driver behavior monitoring since that the monitoring results may be affected by subjective judgments. The physiological signals show higher capability of detecting driver fatigue as it solely depends on the drivers condition. Patel et al. ${ }^{3}$ presented an artificial intelligence based system that could detect early onset of fatigue in drivers using heart rate variability (HRV) as the human physiological measure. $\mathrm{Fu}$ et al. $^{4}$ investigated noncontact sensi- 
tive measurement of driver fatigue during a continuous driving based on EMG and ECG. Recently, a number of methods have been reported in literature for detecting fatigue by using EEG signals, due to the fact that EEG is closely associated with mental and physical activities. Chai et al. ${ }^{5}$ presented a two-class electroencephalography-based method for classifying driver fatigue from EEG recordings. Hajinoroozi et al. ${ }^{6}$ proposed a novel channel-wise convolutional neural network (CCNN) for prediction of driver's cognitive states from EEG signals. Huang et al. ${ }^{7}$ used an online closed-loop EEG-based fatigue detection system to predict driving fatigue based on EEG power spectra. Wang et al. ${ }^{8}$ used wavelet entropy with a sliding window and pulse coupled neural network (PCNN) to detect potential danger during fatigue driving. Driving fatigue is a complex phenomenon involving many physical behavioral processes, in spite of the various methods proposed by researchers, the mechanisms underlying driving mental fatigue still require further investigation. The analysis of EEG signals has become an important topic in recent years. Notably, various EEG-based analysis have been successfully applied to investigate major depressive disorder, ${ }^{9,10}$ autism spectrum disorder, ${ }^{11}$ and seizure detection, ${ }^{12,13}$ and fatigue. Many studies have suggested that the brain can be viewed as a complex network system. ${ }^{14-30}$ In this paper, we develop a multiplex LPHVG theory and construct weighted brain networks to investigate how the topology of the brain functional network is influenced by driving mental fatigue.

In recent years, a new multidisciplinary methodology using complex network has emerged for characterizing complex systems, ${ }^{31-36}$ especially the complex network analysis of time series has undergone a dramatic advance. ${ }^{37-47}$ In particular, Lacasa et al. proposed the (horizontal) visibility graph ${ }^{39,40}$ and the multiplex visibility graph, ${ }^{48}$ which allow mapping a time series into a complex network. The visibility graph theory has been proved to be computationally efficient and an analytically tractable method, which allows probing the dynamics underlying real complex systems from time series. ${ }^{12,49-51}$ More recently, we extended the visibility graph theory to develop multiscale limited penetrable horizontal visibility graph (MLPHVG). ${ }^{52}$ Our proposed methods have been successfully applied in the analysis of signals from an electromechanical system in process industry, ${ }^{53}$ and EEG signals related to manual acupuncture ${ }^{54}$ and Alzheimer's disease. ${ }^{55}$ Despite all these successes, the range of applicability of visibility methods have been limited to univariate time series to a large extent, whereas the most widely existent in science and engineering are multivariate time series. In this paper, inspired by Lacasa et al.,${ }^{48}$ we develop a multiplex limited penetrable horizontal visibility graph (Multiplex LPHVG) method to analyze multichannel EEG signals recorded from subjects performing simulated driving task. We combine the average edge overlap and network measures including clustering coefficient, global efficiency and characteristic path length to detect driver fatigue. The results demonstrate that our method not only allows the detection of driver fatigue with a high accuracy but also enables to characterize the cognitive processes during the mental fatigue states.

\section{Experiment}

\subsection{Subjects}

Participants for this study are ten right-handed students ( 8 males and 2 females, mean age: 23.3 years; range: $22 \sim 25$ years) recruited from Tianjin University. None of the participants have any psychiatric related disorders. Subjects are required to refrain from anti-fatigue drinks or drowsiness causing medications for two days before the experiment. Concurrently, they need to keep reasonable rest with sleep durations of more than 7 hours per night. The experiments are conducted in indoor driving simulators. As all subjects have no exposure to driving simulators, they are asked to practice driving until skilled. Subjects are advised to stop driving at any moment during the experiment when any discomfort appears.

\subsection{Experimental protocol}

The experiments are conducted in the Laboratory of Complex Networks and Intelligent Systems at Tianjin University. A simple driving simulator is equipped with a brake pedal, a steering wheel, an accelerator and a clutch. A monotonous expressway with few bends, sunny day and a bare roadside scenery is involved during the experiment. Furthermore, we add a webcam 360D618 and a projector for better visual perception and a stereo cabinet. The experimental setup is shown in Fig. 1. 


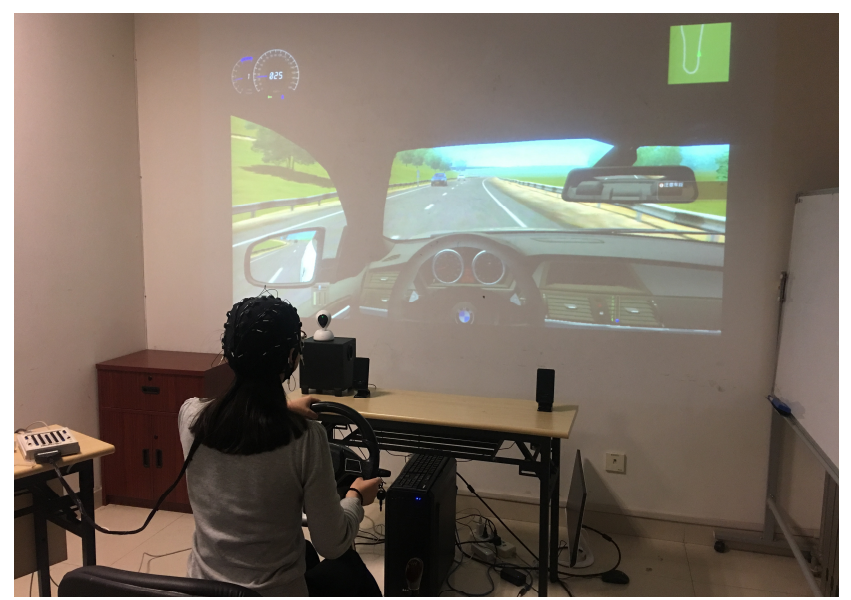

Figure 1. (Color online) The simulating experimental setup for obtaining EEG signals of subjects under alert and fatigue driving states.

For heightening the fatigue sensation of subjects, trials begin during 14:00 15:30, which is proved to be an easy-trapped period for fatigue and each trial lasted approximately 90 minutes. Fullcourse scalp EEG signals of subjects are collected in an isolated and silent room. Besides, we also monitor subjects facial state via a front-facing camera to verify fatigue levels. According to the 9-point Karolinska Sleepiness Scale (KSS) that assesses from 1 (extremely alert) to 9 (very sleepy), drivers fatigue state is divided into alert, mild fatigue and fatigue for assessing subjective fatigue. Since the experiment procedure is a little tiresome, repetitive but mentalengaged, driver fatigue is supposed to increase over time during studies. The subjective assessment and actual behavior, which is an important physiological measurement of driving mental fatigue, reveal that the participants are alert before the task and are fatigued after the task.

\subsection{Data acquisition and preprocessing}

As a measurement of drivers fatigue state, their scalp EEG signals are collected by a 40-channel recording cap (Neuroscan, America) at a sampling frequency of $1000 \mathrm{~Hz}$. The electrodes are arranged in accordance to the standard international 10/20 system. Before acquisition, the skin impedance of EEG electrodes is adjusted below $5 k \Omega$ by injecting conductive gel. The left and right mastoids are defined as reference electrodes. Eye movements and blinking are monitored by recording the horizontal and vertical EOG. All subjects are advised to possibly restrict unnecessary body movement and try to maintain constant speed and avoid car collision during data collection. After the EEG signals are collected, the main steps of data preprocessing are carried out by the EEGLAB software. The raw signals are down-sampled to $250 \mathrm{~Hz}$ and altered using a band-pass FIR filter of $1-50 \mathrm{~Hz}$. Then the Independent Component Analysis (ICA) ${ }^{56}$ is applied to remove the eye blinking artifacts from the datasets by rejecting the highly correlated components with EOG.

\section{Method}

For a multi-channel EEG signal $\left\{x_{\alpha, i}\right\}_{i=1}^{N}, \alpha=$ $1,2, \ldots, M$ containing $M$ channels of EEG signals of equal length $\mathrm{N}$, the Multiplex LPHVG method can be implemented by the following three main steps:

Firstly, the limited penetrable horizontal visibility graph for each EEG channel signal is constructed. A schematic diagram is shown in Fig. 2 to show how to infer limited penetrable horizontal visibility graph from a time series of length 10 . The time series is displayed in the form of vertical bars in Fig.2(a) and each data point (vertical bar) is regarded as a node of a complex network. For the horizontal visibility graph, two nodes $y(i)$ and $y(j)$ are connected if one can draw a horizontal line joining $y(i)$ and $y(j)$ that does not intersect any intermediate data height. That is, a connection between two nodes $y(i)$ and $y(j)$ exists (black lines in Fig.2(b)) if the following criterion is fulfilled:

$$
y(i), y(j)>y(k) \quad \forall i<k<j
$$

The limited penetrable horizontal visibility graph is a development of the HVG. If we set the limited penetrable distance to $\mathrm{L}$, a connection between two nodes exists if the number of in-between nodes that block the horizontal line is no more than L. As shown in Fig.2(b), the red lines are the new established connections when we infer the LPHVG on the basis of HVG with the limited penetrable distance being 1 . 


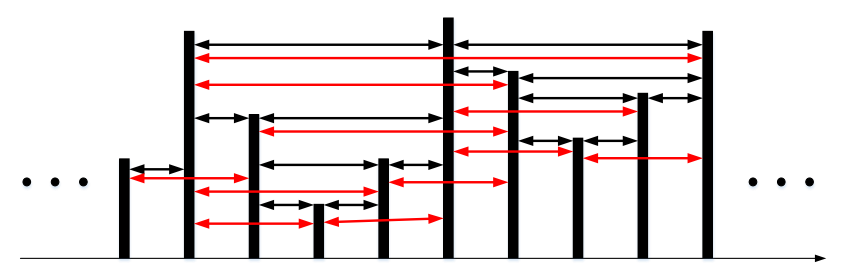

(a)

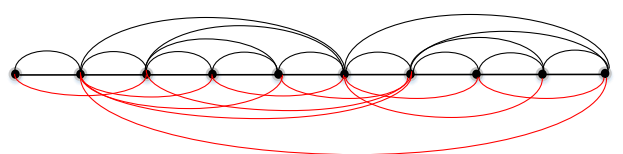

(b)

Figure 2. (Color online) Example of (a) a time series and (b) its corresponding LPHVG with the limited penetrable distance $\mathrm{L}$ being 1 , where every node corresponds to time series data in the same order.

Secondly, we obtain M limited penetrable horizontal visibility graphs $\left\{A^{\alpha}\right\}_{\alpha=1}^{M}$ for multi-channel EEG signal $\left\{x_{\alpha, i}\right\}_{i=1}^{N}, \alpha=1,2, \ldots, M$.The obtained $M$-layer multiplex network on $\mathrm{N}$ nodes is defined by the vector of adjacency matrices, where $A^{\alpha}$ is the $\alpha$ th layer (i.e. layer $\alpha$ ) and corresponds to the LPHVG associated to the EEG channel of $\left\{x_{\alpha, i}\right\}_{i=1}^{N}$. According to Ref. [48], we use the average edge overlap to calculate the overall coherence of the original multivariate time series, which can be calculated as follows:

$$
\omega=\frac{\sum_{i} \sum_{j>i} \sum_{\alpha} a_{i j}^{[\alpha]}}{M \sum_{i} \sum_{j>i}\left(1-\delta_{0, \sum_{\alpha} a_{i j}^{[\alpha]}}\right)}
$$

where $\delta$ is the Kronecker delta symbol, $a_{i j}^{[\alpha]}$ is the edge between nodes $i$ and $j$ at layer $\alpha$. This measure represents the average number of identical edges over all layers of the multiplex network. $\omega$ estimates the similarity and coherence with averaged existence of overlapped links from all pairs of nodes between all layers. $\omega$ takes values in $[1 / M, 1] . \omega=1 / M$ if each edge $i, j$ exists in exactly one layer; $\omega=1$ only if all the $M$ layers are identical.

Thirdly, for a M-layer multiplex network $\left\{A^{\alpha}\right\}_{\alpha=1}^{M}$,we infer the weighted brain network by quantifying the interlayer correlations between the degrees of the same node at two different layers.

$$
k_{i}^{\alpha}=\sum_{j=1}^{N} a_{i j}^{\alpha}
$$

$k_{i}^{\alpha}=\sum_{j=1}^{N} a_{i j}$ is the degree of node $i$ at layer $\alpha$. $p\left(k^{\alpha}\right)$ is the degree distribution of the $\alpha$-th layer,i.e., $p\left(k^{\alpha}\right)$ is a probability that a node has a degree of $k^{\alpha}$ at layer $\alpha$. We characterize the interlayer correlation between two layers $\alpha$ and $\beta$ by using the mutual information of the corresponding degree sequences $k_{i}^{\alpha}$ and $k_{i}^{\beta}, i=1, \ldots, N$. We compute the quantities:

$$
p\left(k^{\alpha}, k^{\beta}\right)=\frac{N_{k^{\alpha}, k^{\beta}}}{N}
$$

where $N_{k^{\alpha}, k^{\beta}}$ is the number of nodes having degree equal to $k^{\alpha}$ and $k^{\beta}$ respectively at layer $\alpha$ and at layer $\beta$. The interlayer correlation of layers $\alpha$ and $\beta$ is: ${ }^{48}$

$$
I_{\alpha, \beta}=\sum_{k^{\alpha}} \sum_{k^{\beta}} p\left(k^{\alpha}, k^{\beta}\right) \log \frac{p\left(k^{\alpha}, k^{\beta}\right)}{p\left(k^{\alpha}\right) p\left(k^{\beta}\right)}
$$

We then construct a brain network by regarding each layer as a node and determining the functional connectivity by calculating the interlayer correlation of all paired layers.

We in this paper develop a multiplex limited penetrable horizontal visibility graph and construct brain network from multi-channel EEG signal for detecting driver fatigue. The three main steps of the method are shown in Fig. 3. The 3D surface visualizations of the weighted brain network is implemented using the Brain Net Viewer. ${ }^{57}$

For all subjects performing the simulated driving task, we define two types of mental state within the 90-min EEG recordings: the first 5 min of the 90min virtual driving operation is defined as the alert state, and the last $5 \mathrm{~min}$ of EEG signals of the 90min virtual driving operation is defined as the fatigue state. The continuous 5 min EEG signals are segmented into 5s non-overlapped epochs, and then we obtain 60 alert epochs and 60 fatigue epochs. 120 multiplex LPHVGs from the alert and fatigue driving EEG signals are constructed with the limited penetrable distance being 1 . The average edge overlap is used to calculate the overall coherence of the original multivariate time series.

Once we obtain the functional brain network, a wide range of sparsity $(10 \% \leq S \leq 35 \%)$ with an interval of $1 \%$ will be applied in all networks to enable comparison of the network statistical measures without bias from different number of edges and also avoid biases associated with using a single threshold. Sparsity $(S)$ is defined as the ratio of the number of existing edges to the max- 

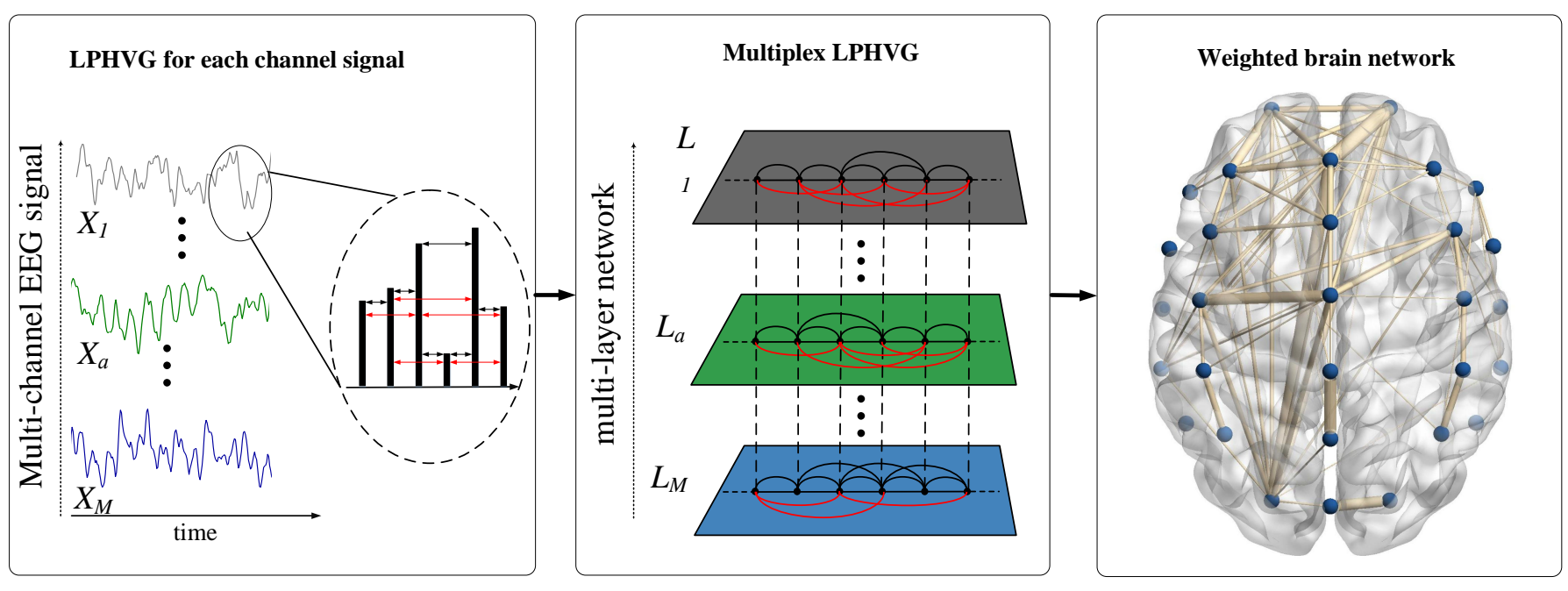

Figure 3. (Color online) Schematic procedures of the multiplex limited penetrable horizontal visibility graph method.

imum possible edge number in the network. And the range of thresholds is selected to ensure that the brain networks maintain small-worldness character within the variation range. ${ }^{58,59}$ We then explore the dynamic behaviors in terms of network analysis, which can be quantified via network statistical measures. An individual network measure may characterize one or several aspects of global and local brain connectivity. In this work, we employ the weighted global efficiency $\left(E^{W}\right)$, weighted clustering coefficient $\left(C^{W}\right)$, and weighted characteristic path length $\left(L^{W}\right)$ to characterize the topological structure of inferred brain networks. ${ }^{60}$ Specifically, These network measures can be calculated as follows:

$$
\begin{aligned}
& \left\{\begin{array}{l}
E^{W}=\frac{\sum_{i \in N} E_{i}^{W}}{n} \\
E_{i}^{W}=\frac{\sum_{j \in N, j \neq i}\left(d_{i j}^{W}\right)^{-1}}{n-1}
\end{array}\right. \\
& \left\{\begin{array}{l}
C^{W}=\frac{\sum_{i \in N} C_{i}^{W}}{n} \\
C_{i}^{W}=\frac{\sum_{j, k \neq i \in N}\left(w_{i j} w_{k j} w_{i k}\right)^{\frac{1}{3}}}{k_{i}\left(k_{i}-1\right)}
\end{array}\right. \\
& \left\{\begin{array}{l}
L^{W}=\frac{\sum_{i \in N} L_{i}^{W}}{n} \\
L_{i}^{W}=\frac{\sum_{j \neq i \in N} d_{i j}}{n-1}
\end{array}\right.
\end{aligned}
$$

where $W$ is short for weighted, since that all the network measures are derived from weighted brain network. $N$ is the set of all nodes in the network, and $n$ is the number of nodes. $E_{i}^{W}$ is the weighted efficiency of node $i . d_{i j}$ is the shortest weighted path length between nodes $i$ and $j, C_{i}^{W}$ is the weighted clustering coefficient of node $i . k_{i}$ is the degree of node $i . w_{i j}, w_{i h}$ and $w_{j h}$ represent the weight between node $i$ and $j, i$ and $h, j$ and $h$, respectively. $L_{i}^{W}$ is the average weighted distance between node $i$ and all other nodes.

\section{Results and Discussions}

The paired t-test is employed to compare the network measures (weighted global efficiency, weighted clustering coefficient, weighted characteristic path length, and average edge overlap respectively) between alert and fatigue driving states of each subject. The paired t-test returns a test decision for the null hypothesis that the network measure in alert and fatigue driving states comes from a normal distribution with mean equal to zero and unknown variance. We show the results in Fig. 4-7. For different subjects, the p-values are all much smaller than 0.05 indicating statistical significance.

We combine the weighted global efficiency, weighted clustering coefficient, weighted characteristic path length and average edge overlap to generate a four-dimensional feature vector and then employ SVM (Support Vector Machine) to realize the classification of alert and mental fatigue driving states. In particular, we employ the leave-one-out crossvalidation to estimate the classified results of the fea- 
tures derived from Multiplex LPHVGs and inferred brain networks. The leave-one-out cross-validation consists of removing one sample from the dataset (alert and mental fatigue), constructing the decision function on the basis only of the remaining dataset and then testing on the removed sample. In this way this process is repeated 120 times independently, with a different sample left out for testing every time. After 120 cross validations, we obtain the predicted labels for all samples and measure the fraction of correctly predicted samples over the total number of samples in the dataset. The classification accuracy, sensitivity and specificity of each subject are shown in Table 1.

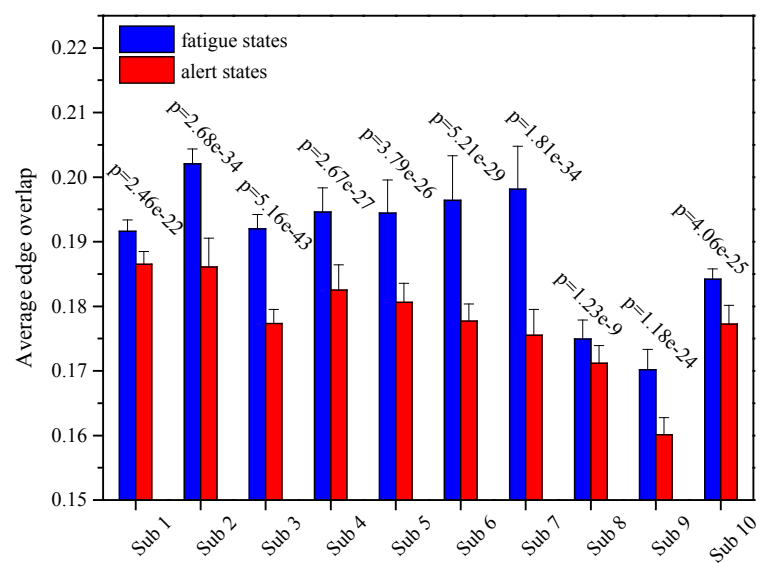

Figure 4. (Color online) The average edge overlap under alert and mental fatigue driving states for different subjects.

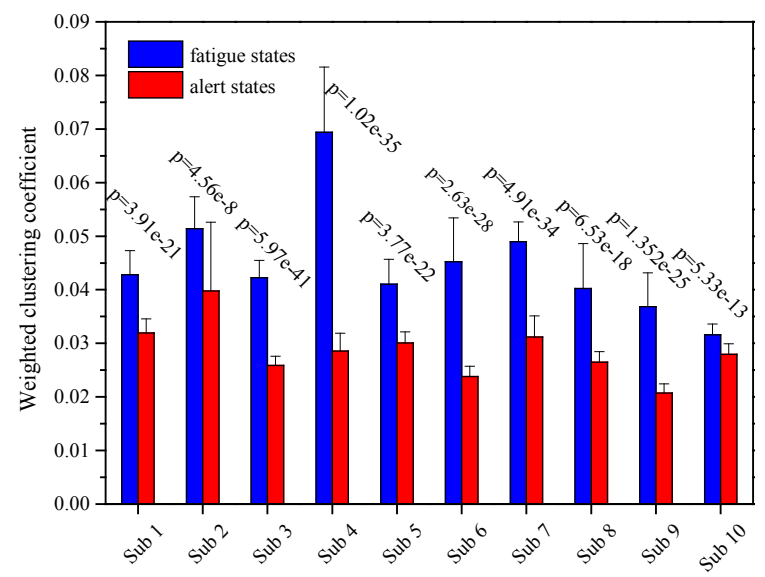

Figure 5. (Color online) The weighted clustering coefficient under alert and mental fatigue driving states for different subjects

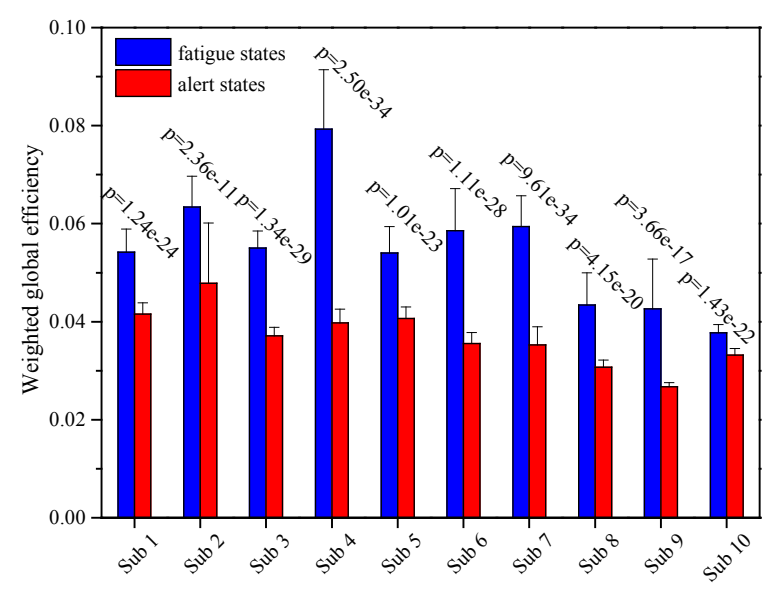

Figure 6. (Color online) The weighted global efficiency under alert and mental fatigue driving states for different subjects.

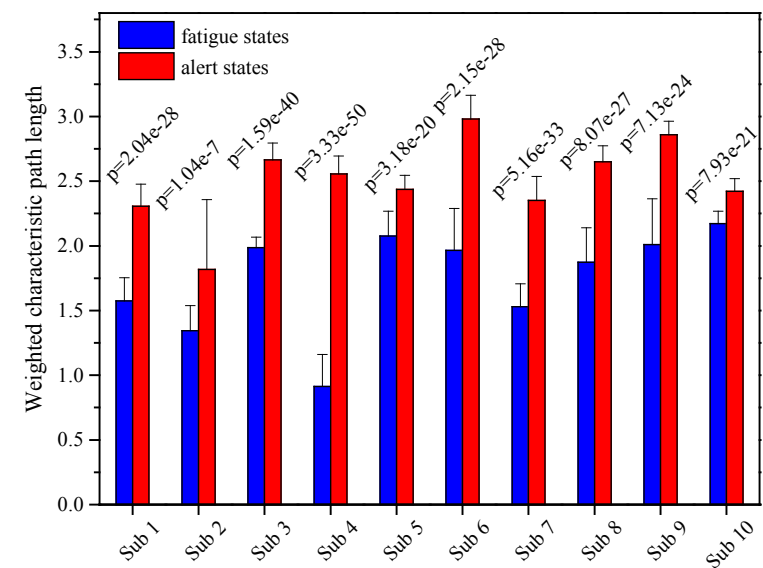

Figure 7. (Color online) The weighted characteristic path length under alert and mental fatigue driving states for different subjects

Table 1. Classification Accuracy, Sensitivity and Specificity of alert and mental fatigue driving states for each subject.

\begin{tabular}{lccc}
\hline & Classification Accuracy & Sensitivity & Specificity \\
\hline Subject 1 & $100 \%$ & $100 \%$ & $100 \%$ \\
Subject 2 & $99.17 \%$ & $98.33 \%$ & $100 \%$ \\
Subject 3 & $100 \%$ & $100 \%$ & $100 \%$ \\
Subject 4 & $100 \%$ & $100 \%$ & $100 \%$ \\
Subject 5 & $96.7 \%$ & $98.28 \%$ & $95.16 \%$ \\
Subject 6 & $98.33 \%$ & $98.33 \%$ & $98.33 \%$ \\
Subject 7 & $100 \%$ & $100 \%$ & $100 \%$ \\
Subject 8 & $97.5 \%$ & $100 \%$ & $95.24 \%$ \\
Subject 9 & $100 \%$ & $100 \%$ & $100 \%$ \\
Subject 10 & $99.17 \%$ & $100 \%$ & $98.33 \%$ \\
\hline
\end{tabular}



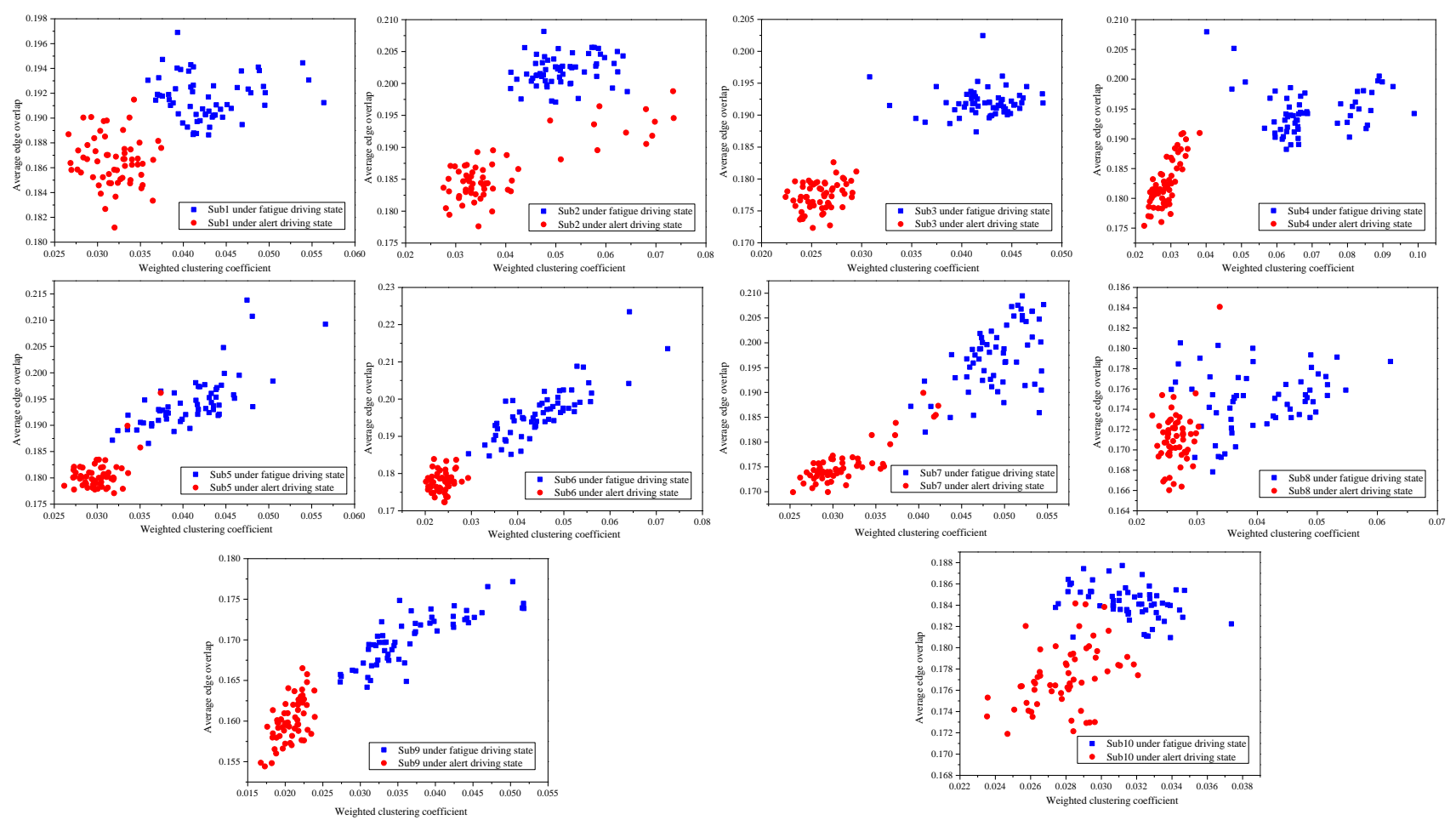

Figure 8. (Color online) The joint distributions of weighted clustering coefficient and average edge overlap for classifying between alert and fatigue driving states of different subjects.

In Fig. 8, we illustrate the joint distributions of weighted clustering coefficient and average edge overlap derived from the 120 epochs (60 alert epochs and 60 fatigue epochs for each subject) of different subjects under alert and fatigue driving states. These measures clearly enable to classify alert and fatigue driving states.

Many EEG-based studies have been performed to detect driver mental fatigue. For example, Wali et al. ${ }^{61}$ obtained $79.21 \%$ accuracy based on fusion of discrete wavelet packet transform (DWPT) and Fast Fourier Transform (FFT). Chai et al. ${ }^{5}$ combined autoregressive (AR) modeling and Bayesian neural network classifier to achieve an accuracy of $88.2 \%$. Correa et al. ${ }^{62}$ got $87.4 \%$ accuracy using a Neural Network classifier. $\mathrm{Li}$ et al. ${ }^{63}$ achieved a classification accuracy of $92.3 \%$ based on Grey Relational Analysis and Kernel Principle Component Analysis. Fu et al. ${ }^{64}$ proposed a dynamic fatigue detection model based on Hidden Markov Model (HMM) to reach a highest accuracy of $95.4 \%$. Min et al. ${ }^{65}$ presented a multiple entropy fusion method to detect a drivers fatigue state by an accuracy of $98.3 \%$. Our proposed multiplex LPHVG method yields an average classification accuracy of $99.09 \%$ over the ten subjects. Except for the high classification accuracy of alert and mental fatigue driving states for different subjects, from Fig. 5 and Fig. 6, we find that the subjects under mental fatigue state had significantly higher clustering coefficients and higher weighted global efficiency. Higher clustering of brain networks is associated with lower wiring $\operatorname{cost}^{66}$ and we can infer that conservation of wiring cost may have been an important selection factor in the evolution of drivers mental state in the simulated driving experiment. Actually, the clustering coefficient has a connection with the local efficiency of information transfer. The mental fatigue can lead to an increase of brain regional synchronous activities, which can be reflected by the increased clustering coefficient. These also account for the reason why the clustering coefficients increase as the brain evolves from alert state to mental fatigue state. ${ }^{67}$ Sengupta et al. ${ }^{68}$ have indicated that an increasing trend in the clustering coefficient is indicative of tight coupling between the corresponding electrode regions that increases in successive stages with 
an increase in fatigue. These existing results support well our interesting findings in the characterization of driving fatigue. In this regard, our method has a capability to explore the variety of brain dynamical properties from alert state to mental fatigue state in the driving process.

\section{Conclusions}

In summary, we have articulated a multiplex limited penetrable horizontal visibility graph strategy for detecting fatigue driving from EEG signals. The proposed method enriches the theories for identifying and characterizing EEG signals recorded from different brain states. The basic procedure are in two parts: First, we construct multiplex limited penetrable horizontal visibility graphs and then extract the average edge overlap. Second, we exploit mutual information theory to infer brain network, where each EEG channel signal is deemed as a node and the edge is determined in terms of interlayer mutual information between the corresponding degree sequences. Combining average edge overlap and network statistical measures, including clustering coefficient and global efficiency, we efficiently classify EEG data recorded from alert and fatigue driving states. In addition, we characterize fatigue behavior from the perspective of complex network, which plays an important role in understanding mental fatigue mechanisms. The results from this study provide means for the development of driver fatigue detection method that could be employed in a real driving context.

\section{Acknowledgements}

This work was supported by National Natural Science Foundation of China under Grant Nos. 61473203, 61873181 and the Natural Science Foundation of Tianjin, China under Grant No. 16JCYBJC18200.

\section{References}

1. S. M. Marcora, W. Staiano and V. Manning, Mental fatigue impairs physical performance in humans, $J$. Appl. Physiol. 106 (3) (2009) 857-864.

2. S. K. L. Lal and A. Craig, A critical review of the psychophysiology of driver fatigue, Biol. Psychol. 55 (3) (2001) 173-194.

3. M. Patel, S. K. L. Lal, D. Kavanagh and P. Rossiter, Applying neural network analysis on heart rate variability data to assess driver fatigue, Expert Syst. Appl. 38 (6) (2011) 7235-7242.
4. R. Fu and H. Wang, Detection of driving fatigue by using noncontact EMG and ECG signals measurement system, Int. J. Neural Syst. 24 (3) (2014) 1450006.

5. R. Chai, G. R. Naik, T. N. Nguyen, S. H. Ling, Y. Tran, A. Craig and H. T. Nguyen, Driver fatigue classification with independent component by entropy rate bound minimization analysis in an EEGBased system, IEEE J. Biomed. Health Inform. 21 (3) (2017) 715-724.

6. M. Hajinoroozi, Z. J. Mao, T. P. Jung, C. T. Lin and Y. F. Huang, EEG-based prediction of driver's cognitive performance by deep convolutional neural network, Signal Process-Image 47 (2016) 549-555.

7. K. C. Huang, T. Y. Huang, C. H. Chuang, J. T. King, Y. K. Wang, C. T. Lin and T. P. Jung, An EEG-Based Fatigue Detection and Mitigation System, Int. J. Neural Syst. 26 (4) (2016) 1650018.

8. H. Wang, C. Zhang, T. Shi, F. Wang and S. Ma, Real-time EEG-based detection of fatigue driving danger for accident prediction, Int. J. Neural Syst. 25 (2) (2015) 1550002.

9. M. Ahmadlou, H. Adeli and A. Adeli. Fractality analysis of frontal brain in major depressive disorder, Int. J. Psychophysiol. 85 (2) (2012) 206-211.

10. M. Ahmadlou, H. Adeli and A. Adeli. Spatiotemporal Analysis of Relative Convergence of EEGs Reveals Differences Between Brain Dynamics of Depressive Women and Men, Clin. EEG Neurosci. 44 (3) (2013) 175-181.

11. M. Ahmadlou, H. Adeli and A. Adeli. Fuzzy Synchronization Likelihood-wavelet methodology for diagnosis of autism spectrum disorder, J. Neurosci. Meth. 211 (2) (2012) 203-209.

12. Z. K. Gao, Q. Cai, Y. X. Yang, N. Dong and S. S. Zhang. Visibility Graph from Adaptive Optimal Kernel Time-Frequency Representation for Classification of Epileptiform EEG, Int. J. Neural Syst. 27 (4) (2017) 1750005.

13. S. Jiang, C. Luo, J. Gong, R. Peng, S. Ma, S. Tan, G. Ye, L. Dong and D. Yao. Aberrant Thalamocortical Connectivity in Juvenile Myoclonic Epilepsy, Int. J. Neural Syst. 28 (1) (2018) 1750034.

14. J. delEtoile, and H. Adeli, Graph theory and brain connectivity in Alzheimer's disease. Neuroscientist 23 (6) (2017) 616-626

15. J. Zhang, W. Cheng, Z. W. Liu, K. Zhang, X. Lei, Y. Yao, B. Becker, Y. C. Liu, K. M. Kendrick, G. M. Lu and J. F. Feng. Neural, electrophysiological and anatomical basis of brain-network variability and its characteristic changes in mental disorders, Brain 139 (2016) 2307-2321.

16. M. Ahmadlou and H. Adeli. Functional community analysis of brain: A new approach for EEG-based investigation of the brain pathology, Neuroimage $\mathbf{5 8}$ (2) (2011) 401-408

17. M. Ahmadlou and H. Adeli. Complexity of weighted graph: A new technique to investigate structural 
complexity of brain activities with applications to aging and autism, Neurosci. Lett. 650 (2017) 103108.

18. E. O'Sullivan-Greene, L. Kuhlmann, E. S. Nurse, D. R. Freestone, D. B. Grayden, M. Cook, A. Burkitt and I. Mareels. Probing to Observe Neural Dynamics Investigated with Networked Kuramoto Oscillators, Int. J. Neural Syst. 27 (1) (2017) 1650038.

19. C. Liu, B. Abu-Jamous, E. Brattico and A. K. Nandi. Towards Tunable Consensus Clustering for Studying Functional Brain Connectivity During Affective Processing, Int. J. Neural Syst. 27 (2) (2017) 1650042.

20. N. Mammone, L. Bonanno, S. De Salvo, S. Marino, P. Bramanti, A. Bramanti and F. C. Morabito. Permutation Disalignment Index as an Indirect, EEGBased, Measure of Brain Connectivity in MCI and AD Patients, Int. J. Neural Syst. 27 (5) (2017) 1750020.

21. M. Grana, L. Ozaeta and D. Chyzhyk. Resting State Effective Connectivity Allows Auditory Hallucination Discrimination, Int. J. Neural Syst. $\mathbf{2 7}$ (5) (2017) 1750019.

22. M. Ahmadlou, H. Adeli and A. Adeli. Graph Theoretical Analysis of Organization of Functional Brain Networks in ADHD, Clin. EEG Neurosci. 43 (1) (2012) 5-13.

23. M. Ahmadlou, A. Adeli, R. Bajo and H. Adeli. Complexity of functional connectivity networks in mild cognitive impairment subjects during a working memory task, Clin. Neurophysiol. 125 (4) (2014) 694-702.

24. J. Gomez-Pilar, J. Poza, A. Bachiller, C. Gomez, P. Nunez, A. Lubeiro, V. Molina and R. Hornero. Quantification of Graph Complexity Based on the Edge Weight Distribution Balance: Application to Brain Networks, Int. J. Neural Syst. 28 (1) (2018) 1750032.

25. C. Schmidt, D. Piper, B. Pester, A. Mierau and H. Witte. Tracking the Reorganization of Module Structure in Time-Varying Weighted Brain Functional Connectivity Networks, Int. J. Neural Syst. 28 (4) (2018) 1750051.

26. G. Galvez, M. Recuero, L. Canuet and F. Del-Pozo. Short-Term Effects of Binaural Beats on EEG Power, Functional Connectivity, Cognition, Gait and Anxiety in Parkinson's Disease, Int. J. Neural Syst. 28 (5) (2018) 1750055.

27. G. Alarcon, D. Jimenez-Jimenez, A. Valentin and D. Martin-Lopez. Characterizing EEG Cortical Dynamics and Connectivity with Responses to Single Pulse Electrical Stimulation (SPES), Int. J. Neural Syst. 28 (6) (2018) 1750057.

28. L. Cheng, Y. Zhu, J. F. Sun, L. F. Deng, N. Y. He, Y. Yang, H. W. Ling, H. Ayaz, Y. Fu and S. B. Tong. Principal States of Dynamic Functional Connectivity Reveal the Link Between Resting-State and TaskState Brain: An fMRI Study, Int. J. Neural Syst. 28 (7) (2018) 1850002.
29. Z. K. Gao, S. Li, Q. Cai, W. D. Dang, Y. X. Yang, C. $\mathrm{X} . \mathrm{Mu}$ and $\mathrm{P}$. Hui, Relative wavelet entropy complex network for improving EEG-based fatigue driving classification, IEEE T. Instrum. Meas. doi: 10.1109/TIM. 2018.2865842

30. Z. K. Gao, K. L. Zhang, W. D. Dang, Y. X. Yang, Z. B. Wang, H. B. Duan and G. R. Chen, An adaptive optimal-Kernel time-frequency representationbased complex network method for characterizing fatigued behavior using the SSVEP-based BCI system, Knowl-Based Syst. 152 (2018) 163-171.

31. N. Liu, H. An, X. Gao, H. Li and X. Hao, Breaking news dissemination in the media via propagation behavior based on complex network theory, Physica $A$ 453 (2016) 44-54.

32. B. Kim, Y. Do and Y. C. Lai, Emergence and scaling of synchronization in moving-agent networks with restrictive interactions, Phys. Rev. E 88 (4) (2013) 042818.

33. W. Zou, D. V. Senthilkumar, R. Nagao, I. Z. Kiss, Y. Tang, A. Koseska, J. Duan and J. Kurths, Restoration of rhythmicity in diffusively coupled dynamical networks, Nat. Commun. 6 (2015) 7709.

34. Z. Wang, S. Kokubo, M. Jusup and J. Tanimoto, Universal scaling for the dilemma strength in evolutionary games, Phys. Life Rev. 14 (2015) 1-30.

35. Z. K. Gao, W. D. Dang, C. X. Mu, Y. X. Yang, S. Li and C. Grebogi, A Novel Multiplex Network-based Sensor Information Fusion Model and Its Application to Industrial Multiphase Flow System, IEEE Trans. Industr. Inform. 14 (9) (2018) 3982-3988.

36. Z. G. Huang, J. Q. Dong, L. Huang and Y. C. Lai, Universal flux-fluctuation law in small systems, Sci. Rep. 4 (2014) 6787.

37. J. Zhang and M. Small, Complex network from pseudoperiodic time series: Topology versus dynamics, Phys. Rev. Lett. 96 (23) (2006) 238701.

38. X. Xu, J. Zhang and M. Small, Superfamily phenomena and motifs of networks induced from time series, PNAS 105 (50) (2008) 19601-19605.

39. L. Lacasa, B. Luque, F. Ballesteros, J. Luque and J. C. Nuno, From time series to complex networks: The visibility graph, PNAS 105 (13) (2008) 4972-4975.

40. B. Luque, L. Lacasa, F. Ballesteros and J. Luque, Horizontal visibility graphs: Exact results for random time series, Phys. Rev. E 80 (4) (2009) 046103.

41. N. Marwan, J. F. Donges, Y. Zou, R. V. Donner and J. Kurths, Complex network approach for recurrence analysis of time series, Phys. Lett. A 373 (46) (2009) 4246-4254.

42. J. F. Donges, J. Heitzig, R. V. Donner and J. Kurths, Analytical framework for recurrence network analysis of time series, Phys. Rev. E 85 (4) (2012) 046105.

43. Z. K. Gao, M. Small and J. Kurths, Complex network analysis of time series, EPL 116 (5) (2016) 50001.

44. Z. K. Gao, S. Li, W. D. Dang, Y. X. Yang, Y. Do and C. Grebogi, Wavelet Multiresolution Complex 
Network for Analyzing Multivariate Nonlinear Time Series, Int. J. Bifurcat. Chaos 27 (8) (2017) 1750123.

45. Z. K. Gao, S. S. Zhang, W. D. Dang, S. Li and Q. Cai, Multilayer Network from Multivariate Time Series for Characterizing Nonlinear Flow Behavior, Int. J. Bifurcat. Chaos 27 (4) (2017) 1750059.

46. W. X. Wang, Y. C. Lai and C. Grebogi, Data based identification and prediction of nonlinear and complex dynamical systems, Phys. Rep. 644 (2016) 1-76

47. W. X. Wang, R. Yang, Y. C. Lai, V. Kovanis and C. Grebogi, Predicting Catastrophes in Nonlinear Dynamical Systems by Compressive Sensing, Phys. Rev. Lett. 106 (15) (2011) 154101.

48. L. Lacasa, V. Nicosia and V. Latora, Network structure of multivariate time series, Sci. Rep. 5 (2015) 15508.

49. M. Ahmadlou, H. Adeli, and A. Adeli, Improved visibility graph fractality with application for the diagnosis of autism spectrum disorder, Physica A 391 (20) (2012) 4720-4726.

50. M. Ahmadlou and H. Adeli, Visibility graph similarity: A new measure of generalized synchronization in coupled dynamic systems, Physica D 241 (4) (2012) 326-332.

51. M. Ahmadlou, H.Adeli, and A. Adeli, New diagnostic EEG markers of the Alzheimers disease using visibility graph, J. Neural Transm. 117 (9) (2010) 1099-1109.

52. Z. K. Gao, Q. Cai, Y. X. Yang, W. D. Dang and S. S. Zhang, Multiscale limited penetrable horizontal visibility graph for analyzing nonlinear time series, Sci. Rep. 6 (2016) 35622.

53. R. Wang, J. Gao, Z. Gao, X. Gao and H. Jiang, Complex network theory-based condition recognition of electromechanical system in process industry, Sci. China Technol. Sc. 59 (4) (2016) 604-617.

54. X. Pei, J. Wang, B. Deng, X. Wei and H. Yu, WLPVG approach to the analysis of EEG-based functional brain network under manual acupuncture, Cogn. Neurodynamics 8 (5) (2014) 417-428.

55. J. Wang, C. Yang, R. Wang, H. Yu, Y. Cao and J. Liu, Functional brain networks in Alzheimer's disease: EEG analysis based on limited penetrable visibility graph and phase space method, Physica A 460 (2016) 174-187.

56. T. P. Jung, S. Makeig, C. Humphries, T. W. Lee, M. J. McKeown, V. Iragui and T. J. Sejnowski. Removing electroencephalographic artifacts by blind source separation, Psychophysiology 37 (2) (2000) 163-178.

57. M. R. Xia, J. H. Wang and Y. He, BrainNet Viewer: A Network Visualization Tool for Human Brain Connectomics, PLoS One 8 (7) (2013) e68910.

58. M. S. Korgaonkar, A. Fornito, L. M. Williams and S. M. Grieve. Abnormal structural networks characterize major depressive disorder: a connectome analysis, Biol. Psychiat. 76 (7) (2014) 567-574.

59. J. R. Zhang, J. H. Wang, Q. Z. Wu, W. H. Kuang, X. Q. Huang, Y. He and Q. Y. Gong. Disrupted Brain Connectivity Networks in Drug-Naive, First-Episode Major Depressive Disorder, Biol. Psychiat. 70 (4) (2011) 334-342.

60. M. Rubinov and O. Sporns, Complex network measures of brain connectivity: Uses and interpretations, Neuroimage 52 (3) (2010) 1059-1069.

61. M. K. Wali, M. Murugappan and B. Ahmmad. Wavelet Packet Transform Based Driver Distraction Level Classification Using EEG, Math. Probl. Eng (2013) 297587.

62. A. G. Correa, L. Orosco and E. Laciar. Automatic detection of drowsiness in EEG records based on multimodal analysis, Med. Eng. Phys. 36 (2) (2014) 244-249.

63. W. Li, Q. C. He, X. M. Fan and Z. M. Fei. Evaluation of driver fatigue on two channels of EEG data, Neurosci. Lett. 506 (2) (2012) 235-239.

64. R. R. Fu, H. Wang and W. B. Zhao. Dynamic driver fatigue detection using hidden Markov model in real driving condition, Expert Syst. Appl. 63 (2016) 397411.

65. J. Min, P. Wang and J. Hu, Driver fatigue detection through multiple entropy fusion analysis in an EEGbased system, PLoS One 12 (12) (2017) e0188756.

66. M. G. Kitzbichler, R. N. A. Henson, M. L. Smith, P. J. Nathan and E. T. Bullmore. Cognitive Effort Drives Workspace Configuration of Human Brain Functional Networks, J. Neurosci. 31 (22) (2011) 8259-8270.

67. Z. Chunlin, Z. Min, Y. Yong, G. Junfeng, R. Nini and L. Pan, The Reorganization of Human Brain Networks Modulated by Driving Mental Fatigue, IEEE J. Biomed. Health Inform. 21 (3) (2017) 743-755.

68. A. Sengupta, A. Routray and S. Kar, Estimation of Fatigue in Drivers by Analysis of Brain Networks,2014 Fourth International Conference of Emerging Applications of Information Technology, 14 (2014) 289-293. 